\title{
Holographic Complexity
}

\author{
Mohsen Alishahiha \\ School of physics, Institute for Research in Fundamental Sciences (IPM) \\ P.O. Box 19395-5531, Tehran, Iran \\ email: alishah@ipm.ir
}

\begin{abstract}
For a field theory with a gravitational dual, following Susskind's proposal we define holographic complexity for a subsystem. The holographic complexity is proportional to the volume of a codimension one time slice in the bulk geometry enclosed by the extremal co-dimension two hypersurface appearing in the computation of the holographic entanglement entropy. The proportionally constant, up to a numerical order of one factor is $G R$ where $G$ is the Newton constant and $R$ is the curvature of the space time. We study this quantity in certain holographic models. We also explore a possible relation between the defined quantity and fidelity appearing in quantum information literature.
\end{abstract}

PACS numbers: $11.25 . \mathrm{Tq}$

\section{INTRODUCTION}

AdS/CFT correspondence [1] as a concrete realization of holographic principle [2, 3] could provide a framework to study quantum gravity and black hole physics. On the other hand theoretical quantum information may also provide a useful tool to study physics of black holes in gravitational theories. Therefore it would be interesting or might even be crucial to understand quantum information holographically, in the sense that for any quantity in quantum information one could have a holographic dual description.

Holographic entanglement entropy [4] is an explicit example in this paradigm which is found useful to study quantum entanglement that might be eventually used to understand the nature of the space time geometry. In quantum information there are other quantities, such as $n$-partite information, which might be of interest from holography point of view. Actually $n$-partite information has been also studied holographically in recent years (see e.g. [5]).

We note, however, that even if we could compute entanglement entropy or in general $n$-partite information (directly or holographically), it might not be enough to fully understand the system under consideration quantum mechanically. It is because no matter which entanglement measure is being computed, we may lose some information of the system simply because the whole system is not a sum of the subsystems.

Actually it was recently pointed out that in order to understand properties of black hole horizons, it is also essential to consider quantum complexity [6]. Suppose our system is in a given state and we would like to map it to another state. Then, intuitively, the complexity tells us that how difficult this task is. In fact, it was conjectured that for an eternal black hole the complexity is proportional to the spatial volume of the Einstein-Rosen bridge connecting two boundaries [7].

Motivated by holographic entanglement entropy and quantum complexity, in this paper we would like to further explore a holographic description of complexity within the context of AdS/CFT correspondence.

To proceed, let us consider a field theory whose holographic dual may be provided by an Einstein gravity on an asymptotically AdS geometry. In this context holographic entanglement entropy for a subsystem in the dual field theory can be computed by minimizing the area of a co-dimension two hyper-surface in the bulk geometry.

More precisely, consider a subsystem $A$ in a time slice in the boundary theory. There is a minimal co-dimension two hyper-surface in the bulk, denoted by $\gamma(A)$, whose boundary coincides with the boundary of the subsystem $\partial \gamma=\partial A$. Then the holographic entanglement entropy is the area of the minimal surface divided by the Newton constant [4]

$$
S_{\mathrm{EE}}=\frac{\operatorname{Area}(\gamma)}{4 G}
$$

Based on this prescription here is an observation. Indeed the way the holographic entanglement entropy is computed would naturally define, rather uniquely, another quantity on the gravity side. Actually the minimal hyper-surface considered above divides a constant time slice into two parts, whose regularized volumes are fixed as soon as the minimal hyper-surface is determined. Therefore beside its area, the volume enclosed by the minimal hyper-surface may also define a new quantity.

To be precise, for a subsystem $A$ in the boundary theory, let us denote by $V(\gamma)$ the volume of the part in the bulk geometry enclosed by the minimal hyper-surface appearing in the computation of entanglement entropy. The corresponding part also contains the subsystem $A$ itself. Then motivated by [6] one may define holographic complexity as follows

$$
\mathcal{C}_{A}=\frac{V(\gamma)}{8 \pi R G},
$$

where $R$ is the radius of the curvature of the space-time, e.g. AdS radius. The numerical factor $8 \pi$ is just a conventional factor. Clearly the definition is ambiguous up 
to an order of one numerical factor. It is also inherently divergent and should be regularized by a UV cut off. Note also that by definition

$$
\mathcal{C}_{A}+\mathcal{C}_{\bar{A}} \leq V_{t s}
$$

where $\bar{A}$ is the complement of $A$ and $V_{t s}$ is the whole regularized volume of the time slice. This inequality saturates for the ground state 1 .

Here we have implicitly assumed that the background is static, though there is a natural generalization for time dependent geometries. Indeed, following the covariant conjecture of holographic entanglement entropy [8] in order to define the corresponding holographic complexity one should compute the volume of a part of the space time enclosed by the extremal co-dimension two hypersurface appearing in the computation of the covariant holographic entanglement entropy 2 .

It is worth noting that in the context of entanglement renormalization [9] the entanglement entropy may be estimated by the minimum number of bonds cut along a curve [10] which could be thought of as Ryu-Takayanagi (RT) curve. Therefore, based on our definition of (2), in this context the holographic complexity may be related (or estimated by) to the number of nods in the area enclosed by the curve cutting the bonds. Such a relation has also been suggested in [7]. Using a holographic model, it might be possible to make this statement more precise [11].

The aim of this paper is to examine the quantity defined in the equation (2) for a certain holographic model. To be concrete we will consider a $d+1$ dimensional CFT in its ground state whose dual description is given by a gravity on an $A d S_{d+2}$ geometry. By making use of the gravity dual we will compute the holographic complexity. It is then natural to look for a proper quantity in the field theory, or in quantum information literature, which could be identified as a holographic dual of the holographic complexity. Actually we will argue that fidelity defined in quantum information might provide such a dual quantity.

The paper is organized as follows. In the next section using an AdS geometry we will compute holographic complexity for a subsystem in the form of a sphere in a strongly coupled CFT. In section three we will compare the results obtained in the section two with fidelity defined for two states in a CFT. The last section is devoted to discussions.

1 Note that one could also define another quantity in terms of the volume as $\mathcal{B}_{A}=\frac{\operatorname{Max}\left\{V_{A}, V_{\bar{A}}\right\}}{8 \pi R G}$, which for the ground state one has $\mathcal{B}_{A}=\mathcal{B}_{\bar{A}}$. Here $V_{A}\left(V_{\bar{A}}\right)$ is the volume in the bulk associated to the subsystem $A(\bar{A})$. Of course this is not the quantity we will consider in this paper. I would like to thank K. Papadodimas for a comment on this point.

2 Using the holographic description of mutual information, one could obviously generalize the holographic complexity for multi subsystems.

\section{HOLOGRAPHIC COMPUTATIONS}

Consider a gravitational theory on an $\mathrm{AdS}_{d+2}$ geometry which could provide a holographic dual for a $d+1$ dimensional strongly coupled CFT in its ground state. Using RT prescription [4] one may compute holographic entanglement entropy for a sphere with radius $\ell$. To do so, it is more convenient to take the following parametrization for the AdS geometry

$$
d s^{2}=\frac{R^{2}}{r^{2}}\left(-d t^{2}+d r^{2}+d \rho^{2}+\rho^{2} d \Omega_{d-1}^{2}\right) .
$$

Then the entangling region is given by $t=$ fixed, $\rho \leq \ell$. To compute the holographic entanglement entropy one needs to minimize the area of a co-dimension two hypersurface in the bulk which may be parametrized by $\rho=$ $f(r)$. It is easy to see that the area is minimized for $f(r)=\sqrt{\ell^{2}-r^{2}}[4]$.

Following our proposal one needs to evaluate the volume enclosed by the above minimal area

$$
\begin{aligned}
V & =\Omega_{d-1} R^{d+1} \int_{\rho \leq f(r)} d \rho d r \frac{\rho^{d-1}}{r^{d+1}} \\
& =\frac{\Omega_{d-1} R^{d+1}}{d} \int_{\varepsilon}^{\ell} d r \frac{\left(\ell^{2}-r^{2}\right)^{d / 2}}{r^{d+1}},
\end{aligned}
$$

where $\Omega_{d-1}$ is the volume of the unit sphere $S^{d-1}$ and $\varepsilon$ should be thought of as a UV cut off. It is easy to perform the integration over $r$ to find the holographic complexity. Indeed for even dimensional CFT's (odd $d$ in our notation) one arrives at

$$
\begin{aligned}
\mathcal{C}_{A}=\frac{\Omega_{d-1} R^{d}}{8 d \pi G}( & \frac{1}{d} \frac{\ell^{d}}{\varepsilon^{d}}-\frac{d}{2(d-2)} \frac{\ell^{d-2}}{\varepsilon^{d-2}}+\frac{d(d-2)}{8(d-4)} \frac{\ell^{d-4}}{\varepsilon^{d-4}} \\
& \left.+\cdots-(-1)^{\left[\frac{d}{2}\right]} \frac{\pi}{2}\right),
\end{aligned}
$$

for $d=1,3,5, \cdots$. Here $[y]$ denotes the integer part of $y$. On the other hand for odd dimensional CFT's (even $d$ ) one gets

$$
\begin{aligned}
\mathcal{C}_{A} & =\frac{\Omega_{1} R^{2}}{16 \pi G}\left(\frac{\ell^{2}}{2 \varepsilon^{2}}-\log \frac{\ell}{\varepsilon}-\frac{1}{2}\right), \\
\mathcal{C}_{A} & =\frac{\Omega_{3} R^{4}}{32 \pi G}\left(\frac{\ell^{4}}{4 \varepsilon^{4}}-\frac{\ell^{2}}{\varepsilon^{2}}+\log \frac{\ell}{\varepsilon}+\frac{3}{4}\right), \\
\mathcal{C}_{A} & =\frac{\Omega_{5} R^{6}}{48 \pi G}\left(\frac{\ell^{6}}{6 \varepsilon^{6}}-\frac{3 \ell^{4}}{4 \varepsilon^{4}}+\frac{3 \ell^{2}}{2 \varepsilon^{2}}-\log \frac{\ell}{\varepsilon}-\frac{11}{12}\right),
\end{aligned}
$$

for $d=2,4,6$, respectively. It is interesting to note that for odd dimensions the holographic complexity contains a logarithmic divergent term.

It is also worth noting that the most divergent term in the expression of holographic complexity is proportional to the volume of the subsystem $V(A)$

$$
\mathcal{C}_{A}=\frac{R^{d}}{8 d \pi G} \frac{V(A)}{\varepsilon^{d}}+\cdots,
$$


leading to a volume law behavior. This should be thought of as an analogous to the celebrated area law of the entanglement entropy. Moreover for arbitrary $d$, the holographic complexity contains a universal term in the sense that it is independent of the UV cut off. For odd $d$ the universal term can be identified with the finite term, while for even $d$ it is given by the coefficient of the log divergence term

$$
\mathcal{C}_{A}^{\text {uni }}=(-1)^{\left[\frac{d}{2}\right]}\left\{\begin{array}{cc}
\frac{\Omega_{d-1} R^{d}}{16 d G} & \text { odd } d, \\
\frac{\Omega_{d-1} R^{d}}{8 d \pi G} & \text { even } d .
\end{array}\right.
$$

Note that the universal terms are also independent of the size of the subsystem $\ell$, indicating that it could reflect certain intrinsic properties of the theory under consideration. In fact it might be thought of as a central charge for the model.

It is also interesting to compute the holographic complexity for a subsystem in an excited state. Holographically an excited state may be described by an asymptotically AdS geometry. To be concrete let us consider an AdS black hole whose metric, adopted to our purpose, may be written as follows

$$
d s^{2}=\frac{R^{2}}{r^{2}}\left(-h(r) d t^{2}+\frac{d r^{2}}{h(r)}+d \rho^{2}+\rho^{2} d \Omega_{d-1}^{2}\right),
$$

where $h(r)=1-m r^{d+1}$ with $m$ being a constant. In this case, similar to the pure AdS background, the minimal hyper-surface in the bulk geometry (9) associated with a sphere subsystem may be parametrized by $\rho=f(r)$, though in the present case the function $f$ does not have a closed simple form. Nonetheless one can find the profile of the minimal surface at leading order in $m$. More precisely assuming $m \ell^{d+1} \ll 1$ one finds 12 .

$f(r)=\sqrt{r_{t}^{2}-r^{2}}\left(1+\frac{2 r_{t}^{d+3}-r^{d+1}\left(r_{t}^{2}+r^{2}\right)}{2(d+2)\left(r_{t}^{2}-r^{2}\right)} m\right)+\mathcal{O}\left(m^{2}\right)$,

where the turning point $r_{t}$ at leading order in $m$ is given by

$$
r_{t}=\ell\left(1-\frac{m \ell^{d+1}}{d+2}+\mathcal{O}\left(\left(m \ell^{d+1}\right)^{2}\right)\right) .
$$

The volume enclosed by the minimal area is

$$
V=\frac{\Omega_{d-1} R^{d+1}}{d} \int_{\varepsilon}^{r_{t}} d r \frac{f(r)^{d}}{r^{d+1} \sqrt{h(r)}} .
$$

The above integral can be evaluated order by order in $m$ and the result would be a function of the turning point $r_{t}$. On the other hand using the expression of the turning point (12) one can re-write the holographic complexity as a function of the radius $\ell$ at leading order in $m$. Doing so, in the present case unlike the entanglement entropy, one finds that at leading order the holographic complexity remains uncharged

$$
\mathcal{C}_{A}^{\mathrm{BH}}=\mathcal{C}_{A}^{\mathrm{AdS}}+\mathcal{O}\left(m^{2}\right) .
$$

In fact one could go further to evaluate the order of $\mathrm{m}^{2}$ term as well. Although the expressions are lengthy, the final result is simple, given by

$$
\Delta \mathcal{C}_{A}=\mathcal{C}_{A}^{\mathrm{BH}}-\mathcal{C}_{A}^{\mathrm{AdS}}=c_{d} \frac{\Omega_{d-1} R^{d}}{8 d \pi G}\left(m \ell^{d+1}\right)^{2},
$$

where $c_{d}$ is a calculable (non-negative) numerical constant. For example one has $c_{1}=0, c_{2}=\frac{1}{128}, \cdots$.

This result may be compared with that of the entanglement entropy where one finds that the variation of entanglement entropy gets corrected at order of $m \ell^{d+1}$ [12, 13]

$$
\Delta S_{\mathrm{EE}}=S_{\mathrm{EE}}^{\mathrm{BH}}-S_{\mathrm{EE}}^{\mathrm{AdS}}=\tilde{c}_{d} \frac{\Omega_{d-1} R^{d}}{G}\left(m \ell^{d+1}\right),
$$

leading to the first law of entanglement 14. Here $\tilde{c}_{d}$ is a numerical factor (see e.g. [12]). Note that $\epsilon=m \ell^{d+1}$ is the expanding parameter which measures how much the ground state is deformed to the excited state. Taking into account that in the present case the energy of the excited state is proportional to $\mathcal{E} \sim \frac{R^{d}}{G} m l^{d}$, up to numerical factors, one arrives at

$$
\begin{aligned}
\Delta S_{\mathrm{EE}} & \sim \frac{R^{d}}{G} \epsilon \sim \mathcal{E} \ell, \\
\Delta \mathcal{C}_{A} & \sim(d-1) \frac{R^{d}}{G} \epsilon^{2} \sim(d-1) \frac{G}{R^{d}} \mathcal{E}^{2} \ell^{2},
\end{aligned}
$$

i.e. while the change of entanglement entropy gets first order correction with respect to energy, the holographic complexity receives second order correction. Here we have explicitly put the factor of $d-1$ to stress that for $d=1$ the correction vanishes.

Finally we should note that although we have done all the computations for a subsystem with spherical symmetry, it could be done for other subsystem such as a strip. An advantage to work with sphere is that we could present the results analytically.

\section{FIDELITY AND HOLOGRAPHIC COMPLEXITY}

The complexity as defined in [6] is a quantity to measure how difficult a task is. Given a quantum system a task would be a unitary evolution to map a state to another state. In quantum information there are several quantities which could provide measures to compare two states. These include, for example, relative entropy or fidelity (see [16] for a holographic description of the relative entropy). The aim of this section is to investigate whether there is any connection between holographic complexity and fidelity.

To explore this point let us start with a quantum pure state $\left|\Psi\left(\lambda_{1}\right)\right\rangle$ in the Hilbert of a quantum system. Where $\lambda$ is a tunable parameter of the model. Now consider a 
neighboring pure state $\left|\Psi\left(\lambda_{2}\right)\right\rangle$ which may be reached by changing, infinitesimally, the parameter $\lambda$. It is then natural to pose a question how close these two states are? To address this question one could compute fidelity 17] which in the present case where both states are pure it is given by the inner product of the two states. For sufficiently small perturbation $\delta \lambda=\lambda_{2}-\lambda_{1}$ one has

$$
\left|\left\langle\Psi\left(\lambda_{1}\right) \mid \Psi\left(\lambda_{2}\right)\right\rangle\right|=1-G_{\lambda} \delta \lambda^{2}+\mathcal{O}\left(\delta^{3}\right),
$$

where $G_{\lambda}$ is fidelity susceptibility. This expression, considered as a metric (see e.g. [18]), could measure the distance between two neighboring quantum pure states.

Recently a gravity dual for information metric was proposed in [15] where it was suggested that under certain approximations the fidelity susceptibility for a $d+1$ dimensional CFT deformed by a marginal perturbation is holographically given by the time slice with the maximal volume in the AdS background which ends on the time slice at the AdS boundary [15].

From our construction it is clear that within the same approximations if one takes infinite volume limit, the holographic complexity reduces to the fidelity susceptibility $G_{\lambda}$ as computed in 15. In particular for a two dimensional CFT from (6) one has

$$
\mathcal{C}_{A}=\frac{c \ell}{12 \pi \varepsilon}-\frac{c}{24}
$$

where $c=\frac{3 R}{2 G}$ is the central charge of the two dimensional CFT. Clearly in the large $\ell$ limit it reduces to that in 15] obtained from AdS Janus solution [19]. It is interesting to note that the finite term in the above expression is proportional to the Casimir energy of the two dimensional CFT which typically appears whenever we are dealing with a CFT in a finite volume. Note that the factor of 24 comes from our particular normalization of $\mathcal{C}_{A}$.

More generally for a $d+1$ dimensional CFT one gets

$$
\left.\mathcal{C}_{A}\right|_{\ell \rightarrow \infty}=\frac{V R^{d}}{8 \pi d G \varepsilon^{d}},
$$

where $V$ is the volume of the time slice in the AdS geometry. This is exactly the fidelity susceptibility obtained in 15] for an AdS background with a defect brane 20. considered as a marginal deformation. It is important to note that to get the right $\frac{1}{c^{d}}$ behavior it is crucial to deform the CFT by an exactly marginal operator [15]. In terms of the information metric $G_{\lambda}$ the inequality (3) reads $\mathcal{C}_{A}+\mathcal{C}_{\bar{A}} \leq G_{\lambda}$.

Note also that the above comparison works just for the extremely large $\ell$ limit. In other words there is, a priori, no way to understand the subleading divergences in this picture, nor it is not clear how to compare the finite temperature case where the temperature dependent term drops in the large $\ell$ limit.

Therefore although this comparison seems reasonable, it is not quite clear to us whether there is a relation between holographic information metric obtained in [15] and the holographic complexity studied in this paper (or that defined in 6] 3 . Nonetheless it might be possible to extend the notation of fidelity susceptibility for a subsystem with a finite size.

To explore this point better it is useful to write the fidelity in terms of a density metric. Denoting by $\varrho\left(\lambda_{1}\right)$ and $\varrho\left(\lambda_{2}\right)$ the density matrices associated with two states $\left|\Psi\left(\lambda_{1}\right)\right\rangle$ and $\left|\Psi\left(\lambda_{2}\right)\right\rangle$ respectively, the fidelity can be written as follows [17]

$$
F=\operatorname{Tr} \sqrt{\sqrt{\varrho\left(\lambda_{1}\right)} \varrho\left(\lambda_{2}\right) \sqrt{\varrho\left(\lambda_{1}\right)}},
$$

which for pure states reduces to the inner product of the states, $\left|\left\langle\Psi\left(\lambda_{1}\right) \mid \Psi\left(\lambda_{2}\right)\right\rangle\right|$. On the other hand dealing with a subsystem, it is natural to consider a reduced density matrix and therefore define the fidelity for two reduced density matrices [21]

$$
F_{A}=\operatorname{Tr}_{A} \sqrt{\sqrt{\varrho_{A}\left(\lambda_{1}\right)} \varrho_{A}\left(\lambda_{2}\right) \sqrt{\varrho_{A}\left(\lambda_{1}\right)}},
$$

where $\varrho_{A}\left(\lambda_{1}\right)$ and $\varrho_{A}\left(\lambda_{2}\right)$ are the corresponding reduced density matrices. Now the aim is to expand the reduced fidelity for a small perturbation to find an expression for reduced fidelity susceptibility.

To proceed, we will take advantage of having a subsystem in the shape of a sphere. Actually when we have a subsystem with spherical symmetry in the ground state of a CFT, one may conformally map the system to a thermal system whose temperature is given by the radius of the sphere; $\beta=2 \pi \ell$ [22]. More precisely, under this conformal map the reduced density matrix maps to a thermal density matrix given by [22]

$$
\varrho_{\mathrm{th}}(\lambda)=\frac{e^{-2 \pi \ell H_{\tau}(\lambda)}}{\operatorname{Tr}\left(e^{-2 \pi \ell H_{\tau}(\lambda)}\right)},
$$

where $H_{\tau}$ is the standard Hamiltonian of the thermal system which corresponds to the time translation. As a result we will have to compute fidelity at finite temperature. Fidelity for a mixed state at finite temperature has been studied in 23] where the authors have considered two possibilities; either to change the temperature while keeping the parameter $\lambda$ fixed, or another way around. In our model since we are dealing with a system with a fixed temperature (fixed $\ell$ ) the corresponding thermal fidelity should be given as follows [23]

$$
F\left(2 \pi \ell, \lambda_{1}, \lambda_{2}\right)=\operatorname{Tr} \sqrt{\sqrt{\varrho_{\mathrm{th}}\left(\lambda_{1}\right)} \varrho_{\mathrm{th}}\left(\lambda_{2}\right) \sqrt{\varrho_{\mathrm{th}}\left(\lambda_{1}\right)}} .
$$

Setting $\lambda_{2}=\lambda_{1}+\delta \lambda$, for sufficiently small $\delta \lambda$ and using the definition of thermal density matrix in terms of the Hamiltonian one gets 24]

$$
F\left(2 \pi \ell, \lambda_{1}, \lambda_{2}\right)=1-2 \pi \ell \chi_{\lambda} \frac{\delta \lambda^{2}}{8}+\mathcal{O}\left(\delta \lambda^{3}\right),
$$

\footnotetext{
${ }^{3}$ I would like to thank T. Takayanagi for a comment on this point.
} 
where $\chi_{\lambda}=\partial_{\lambda}^{2} \mathcal{F}_{\text {th }}$ is the fidelity susceptibility given in terms of the free energy of the thermal system, $\mathcal{F}_{\text {th }}$. If one perturbs the system by an operator with dimension $\Delta$, then the fidelity susceptibility scales as $\frac{R^{d}}{\varepsilon^{2 \Delta-2-d}}$, where $R$ is a scale of the model (see e.g. 25]). Thus for a marginal operator where $\Delta=d+1$ one gets $\chi_{\lambda} \sim(R / \varepsilon)^{d}$. On the other hand the free energy and therefore susceptibilities receive finite temperature corrections which have an expansion in power of $T^{2}$ (see e.g. [26]). Therefore for our mixed thermal state, where the temperature is given by $T=\frac{1}{2 \pi \ell}$, one arrives at

$$
\chi_{\lambda} \sim \frac{R^{d}}{\varepsilon^{d}}\left(1+c_{2} \frac{\varepsilon^{2}}{\ell^{2}}+c_{4} \frac{\varepsilon^{4}}{\ell^{4}}+\cdots\right),
$$

in qualitative agreement with our results in the previous section.

It is also interesting to use the inverse of the conformal map to return to the original picture of the reduced density matrix. In fact doing so, one gets

$$
F_{A}=1-\partial_{\lambda}^{2} \mathcal{F} \frac{\delta \lambda^{2}}{8}+\mathcal{O}\left(\delta \lambda^{3}\right),
$$

where $\mathcal{F}(\lambda)=\operatorname{Tr}\left(\varrho_{A}(\lambda) H(\lambda)\right)-S_{\mathrm{EE}}(\lambda)$ with $S_{\mathrm{EE}}$ being the entanglement entropy and, $H(\lambda)$ is the modular Hamiltonian by which the reduced density matrix may be given as follows

$$
\varrho_{A}(\lambda)=\frac{e^{-H(\lambda)}}{\operatorname{Tr}\left(e^{-H(\lambda)}\right)} .
$$

Note that in terms of the modular Hamiltonian the fidelity susceptibility $\chi=\partial_{\lambda}^{2} \mathcal{F}$ is given by

$$
\chi=\left\langle H^{2}\right\rangle-\langle H\rangle^{2} .
$$

Since the explicit form of the modular Hamiltonian is known for the subsystem we are considering [14], it would be interesting to find the fidelity susceptibility directly from the modular Hamiltonian. We are currently working on this line.

\section{DISCUSSIONS}

In this paper we have defined and studied holographic complexity for a subsystem in a CFT which has a holographic description. Motivated by the holographic entanglement entropy, the corresponding quantity has been defined by the volume enclosed by the extremal codimension two hyper-surface appearing in the computation of the holographic entanglement entropy.

We have also compared the holographic complexity of the ground state of a CFT deformed by a marginal operator with the reduced fidelity susceptibility where we have seen that these two quantities qualitatively behave in a similar manner. We have also noticed that in large volume limit the holographic complexity reduces to holographic information metric studied in [15].

It is also illustrative to apply the above suggestion for the case of thermofield doubled CFT's whose gravitation dual may be provided by an eternal black hole. The entanglement entropy for this model has been studied in [27] where it was shown that the holographic entanglement entropy is given by the area of an extremal surface connecting two boundaries. Following the equation (2), one needs to compute the volume enclosed by this extremal hyper-surface. Actually for this system the complexity has been studied in 7] where it was shown that the complexity is given by the spatial volume of the Einstein-Rosen bridge connecting two boundaries.

It is worth mentioning that through the whole of this paper we have implicitly assumed that the gravitational theory is given by an Einstein gravity. It is then natural to ask how to define holographic complexity when we have a gravitational theory with higher derivative terms? Note that for this case the thermal entropy and holographic entanglement entropy are given by Wald entropy and the generalized entropy functional (see e.g. [28]), respectively. Therefore we would expect to have a general expression (which is not necessary the volume) for the holographic complexity as well.

Actually to address this question one needs to understand two issues. First of all one should understand how to specify the corresponding co-dimension two hypersurface in the bulk. And secondly, even when we have fixed a part of the time slice, what quantity should be evaluated in this time slice?

In fact there is a natural proposal for holographic complexity in a general gravitational theory which is as follows. Consider the Wald charge appearing as the integrand in the Wald formula for entropy. Then evaluate it on a co-dimension one time slice enclosed by a co-dimension two hyper-surface minimizing the entropy functional appearing in the computation of holographic entanglement entropy. More explicitly

$$
\mathcal{C}_{A}=-\frac{1}{R} \int_{\text {enclosed volume }} \frac{\partial \mathcal{L}}{\partial R_{\mu \nu \rho \sigma}} \epsilon^{\mu \nu} \epsilon^{\rho \sigma},
$$

where $\epsilon^{\mu \nu}$ is binormal to the co-dimension two hypersurface enclosing the volume. Here the factor of $\frac{1}{R}$ comes form a dimensional analysis and the fact that the only natural dimensionfull parameter of the model is the curvature radius of the space time. In fact this factor is the same as the extra $R$ appearing in equation (2). Clearly for Einstein gravity the above expression reduces to the volume. On the other hand if one computes the holographic complexity for a sphere in the most general quadratic action

$$
\int d^{d+2} x \sqrt{g}\left(\alpha_{1} R_{\mu \nu \rho \sigma} R^{\mu \nu \rho \sigma}+\alpha_{2} R_{\mu \nu} R^{\mu \nu}+\alpha_{3} R^{2}\right),
$$

then it gets corrected by an overall factor given by $4 \alpha_{1}+2(d+1)\left(\alpha_{2}+(d+2) \alpha_{3}\right)$. Interestingly enough 
it is exactly the factor which appears in the correction of the entanglement entropy for a sphere. It is then natural to think of the universal term of holographic complexity as the central charge of the model (see also (19)).

Clearly this point deserves more investigations. We would like to mention that quantum complexity for a general theory is extensively studied in [29]. Of course it is not clear to us whether, for a generic case, there is a direct relation between quantum complexity studied in 29] and holographic complexity studied in the present paper.

\section{ACKNOWLEDGMENTS}

I would like to thank Luis Alvarez-Gaume and Kyriakos Papadodimas for discussions. The author would also like to thank Vahid Karimipour, Leonard Susskind and Tadashi Takayanagi for correspondences and comments. Special thank to Leonard Susskind for encouragements and providing me a draft of [29] prior to publication. I would also like to thank CERN TH-Division for very warm hospitality. This work is supported by Iranian National Science Fundation (INSF).
[1] J. M. Maldacena, "The Large N limit of superconformal field theories and supergravity, "Int. J. Theor. Phys. 38, 1113 (1999) [Adv. Theor. Math. Phys. 2, 231 (1998)] hep-th/9711200.

[2] G. 't Hooft, "Dimensional reduction in quantum gravity, "Salamfest 1993:0284-296 gr-qc/9310026.

[3] L. Susskind, "The World as a hologram, " J. Math. Phys. 36, 6377 (1995) hep-th/9409089.

[4] S. Ryu and T. Takayanagi, "Holographic derivation of entanglement entropy from AdS/CFT, " Phys. Rev. Lett. 96, 181602 (2006) hep-th/0603001.

[5] M. Alishahiha, M. R. M. Mozaffar and M. R. Tanhayi, "Evolution of Holographic n-partite Information, " arXiv:1406.7677 [hep-th].

[6] L. Susskind, "Computational Complexity and Black Hole Horizons, " arXiv:1403.5695 [hep-th], arXiv:1402.5674 [hep-th].

[7] D. Stanford and L. Susskind, "Complexity and Shock Wave Geometries," Phys. Rev. D 90, no. 12, 126007 (2014) arXiv:1406.2678 [hep-th]].

[8] V. E. Hubeny, M. Rangamani and T. Takayanagi, "A Covariant holographic entanglement entropy proposal, " JHEP 0707 (2007) 062 arXiv:0705.0016 [hep-th]].

[9] G. Vidal, "Entanglement Renormalization," Phys. Rev. Lett. 99, no. 22, 220405 (2007) cond-mat/0512165.

[10] B. Swingle, "Entanglement Renormalization and Holography, " Phys. Rev. D 86, 065007 (2012) arXiv:0905.1317 [cond-mat.str-el]].

[11] M. Alishahiha, Work in progress.

[12] J. Bhattacharya, M. Nozaki, T. Takayanagi and T. Ugajin, "Thermodynamical Property of Entanglement Entropy for Excited States, " Phys. Rev. Lett. 110, no. 9, 091602 (2013) arXiv:1212.1164.

[13] D. Allahbakhshi, M. Alishahiha and A. Naseh, "Entanglement Thermodynamics, " JHEP 1308, 102 (2013) arXiv:1305.2728 [hep-th]].

[14] D. D. Blanco, H. Casini, L. Y. Hung and R. C. Myers, "Relative Entropy and Holography," JHEP 1308, 060 (2013) arXiv:1305.3182 [hep-th]].

[15] M. Miyaji, T. Numasawa, N. Shiba, T. Takayanagi and
K. Watanabe, "Gravity Dual of Quantum Information Metric," arXiv:1507.07555 [hep-th].

[16] J. Lin, M. Marcolli, H. Ooguri and B. Stoica, "Locality of Gravitational Systems from Entanglement of Conformal Field Theories, " Phys. Rev. Lett. 114 (2015) 22, 221601 arXiv:1412.1879 [hep-th]].

[17] A. Uhlmann, "The transition probability in the state space of A*-algebra, " Rep. Math. Phys. 9273 (1976),

[18] S. L. Braunstein and C. M. Caves, "Statistical distance and the geometry of quantum states, " Phys. Rev. Lett. 72, 3439 (1994).

[19] D. Bak, M. Gutperle and S. Hirano, "Three dimensional Janus and time-dependent black holes, " JHEP 0702, 068 (2007) hep-th/0701108.

[20] A. Karch and L. Randall, "Locally localized gravity," JHEP 0105, 008 (2001) hep-th/0011156.

[21] H. Q. Zhou, "Renormalization group flows and quantum phase transitions: fidelity versus entanglement," arXiv:0704.2945.

[22] H. Casini, M. Huerta and R. C. Myers, "Towards a derivation of holographic entanglement entropy, " JHEP 1105, 036 (2011) arXiv:1102.0440 [hep-th]]

[23] P. Zanardi, H.-T. Quan, X.-G. Wang, and C.-P. Sun, "Mixed-state fidelity and quantum criticality at finite temperature," Phys. Rev. A 75, 032109 (2007).

[24] H. T. Quan and F. M. Cucchietti, "Quantum fidelity and thermal phase transitions, " Phys. Rev. E 79, 031101 (2009).

[25] S-J. Gu, Fidelity Approach to Quantum Phase Transi- tions, Int. J. Mod. Phys. B 24 (2010) 4371 arXiv:0811.3127 [quant-ph]].

[26] M. Laine, " Basics of Thermal Field Theory, " 2013, http://www.laine.itp.unibe.ch/basics.pdf

[27] T. Hartman and J. Maldacena, "Time Evolution of Entanglement Entropy from Black Hole Interiors, " JHEP 1305 (2013) 014 [arXiv:1303.1080 [hep-th]].

[28] X. Dong, "Holographic Entanglement Entropy for General Higher Derivative Gravity, " JHEP 1401, 044 (2014) arXiv:1310.5713 [hep-th], arXiv:1310.5713.

[29] A. R. Brown, D. A. Roberts, L. Susskind, B. Swingle and 
Y. Zhao, "Complexity Equals Action," arXiv:1509.07876

[hep-th]. 